\title{
Resonantly Driven Laser-Plasma Electron Accelerators
}

\author{
D. Umstadter, J. Kim, E. Esarey ${ }^{1}$, E. Dodd, and T. Neubert \\ Center for Ultrafast Optical Science* \\ University of Michigan \\ Ann Arbor, MI 48109
}

\begin{abstract}
A method for generating large-amplitude nonlinear plasma waves, which utilizes an optimized train of independently adjustable, intense laser pulses, is analyzed in 1-D both theoretically and numerically (using both Maxwell-fluid and particle-in-cell codes). Optimal pulse widths and interpulse spacings are computed for pulses with either square or finite-risetime sine shapes. A resonant region of the plasma wave phase space is found where the plasma wave is driven by the laser most efficiently. The width of this region, and thus the optimal finiterisetime laser pulse width, was found to decrease with increasing plasma density and plasma wave amplitude, while the nonlinear plasma wavelength, and thus the optimal interpulse spacing, was found to increase. Also investigated are the resonance sensitivities to variations in the laser and plasma parameters. Nonlinear Landau damping of the wave by trapped background electrons is found to be important. Resonant excitation by this method is shown to more advantageous for electron acceleration than either the single pulse wakefield or the plasma beatwave concepts, because comparable plasma wave amplitudes may be generated at lower plasma densities, thus reducing electron-phase detuning, or at lower laser intensities, thus reducing laser-plasma instabilities. Practical experimental methods for producing the required pulse trains are discussed.
\end{abstract}

\section{INTRODUCTION}

The generation of large-amplitude, relativistic plasma waves is a subject of much current interest $^{2-5}$ because of its potential use for ultrahigh-gradient electron acceleration ${ }^{3}$. While conventional rf-driven accelerators are limited to fields $\leq 1 \mathrm{MV} / \mathrm{cm}$, plasma accelerators have been shown experimentally ${ }^{4}$ to support gradients $\leq 100 \mathrm{MV} / \mathrm{cm}$. The maximum axial electric field of a relativistic plasma wave, as predicted by one-dimensional (1-D) cold fiuid theory, is the "wave-breaking" field", $E_{W B}=E_{0} \sqrt{2\left(\gamma_{p}-1\right)}$, where $E_{0}=\left(m_{e} c \omega_{p} / e\right) \simeq$ $0.96 n_{e 0}^{1 / 2}\left[\mathrm{~cm}^{-3}\right] \mathrm{V} / \mathrm{cm}, \omega_{p}=\left(4 \pi e^{2} n_{e 0} / m_{e}\right)^{1 / 2}$ is the electron plasma frequency, $n_{e 0}$ is the

"send inquiries to dpu@dip.eecs.umich.edu. 
ambient electron density, $\gamma_{p}=\left(1-v_{p}^{2} / c^{2}\right)^{-1 / 2}$, and $v_{p}$ is the phase velocity of the plasma wave. For a laser-driven, plasma-based accelerator, $\gamma_{p} \simeq \gamma_{g} \simeq \omega / \omega_{p} \gg 1$, where $\gamma_{g}=(1-$ $\left.v_{g}^{2} / c^{2}\right)^{-1 / 2}, v_{g}$ is the group velocity of the laser, and $\omega$ is the laser frequency. For a laser of wavelength $\lambda \simeq 2 \pi c / \omega=1 \mu \mathrm{m}$ and a plasma of density $n_{e 0}=10^{16} \mathrm{~cm}^{-3}, \gamma_{g} \simeq 300$, $E_{0} \simeq 100 \mathrm{MV} / \mathrm{cm}$ and $E_{W B} \simeq 2.5 \mathrm{GV} / \mathrm{cm}$.

Until recently, only two major types of laser-driven, plasma-based accelerators had been investigated: the plasma beatwave accelerator $(\mathrm{PBWA})^{3,4}$, and the laser wakefield accelerator (LWFA) $)^{3,7}$. In the PBWA, two laser beams of frequencies $\omega_{1}$ and $\omega_{2}$ are optically mixed in a plasma to produce a laser beatwave of frequency $\Delta \omega=\omega_{1}-\omega_{2}$, i.e., in effect a train of fixed equally spaced pulses of equal pulse widths. By adjusting the beat frequency and/or the plasma density such that $\Delta \omega \simeq \omega_{p}$, the laser beatwave can resonantly drive a large amplitude plasma wave. As the plasma wave amplitude grows, however, nonlinear effects cause the wavelength of the plasma wave to increase and, hence, the resonant frequency is shifted away from $\Delta \omega$. Eventually the relative phase between the laser beatwave and the plasma wave becomes $\pi / 2$ out of phase and the beatwave no longer drives the plasma wave to higher amplitudes. This resonant detuning due to the increase in the plasma wavelength ultimately limits the plasma wave amplitude in the PBWA. In the LWFA, the ponderomotive force associated with the gradients in the intensity of a single, ultrashort laser pulse ${ }^{8}$ drives a plasma wave "wake-field." The maximum plasma wave amplitude is obtained when the pulse duration $\tau$ and/or plasma density is adjusted such that $\tau \simeq 2 \pi / \omega_{p}$. The plasma wave amplitude increases as the laser intensity $I$ increases and the laser pulse length decreases. Hence, in the LWFA, the plasma wave amplitude is limited by the maximum laser intensity and the minimum laser pulse length which can be obtained by laser technology. Currently, these values are limited to ${ }^{8} I \sim 10^{18} \mathrm{~W} / \mathrm{cm}^{2}$ and $\tau \sim 50 \mathrm{fs}$.

Recently, the self-modulated LWFA has been suggested ${ }^{9,10}$. Here, a single laser pulse is incident on a plasma with a density that is higher than the "resonant density" such that the laser pulse duration is now several plasma periods, i.e., $\tau>2 \pi / \omega_{p}$. Due to a self-modulation instability ${ }^{11}$, the pulse breaks up into multiple pulses, each of which is "resonant." Although higher plasma densities and the high-intensity multiple-pulse structure lead to higher wakefield amplitudes, they are difficult to achieve simultaneously due to plasma defocusing ${ }^{12}$. In simulations of the self-modulated LWFA ${ }^{9}$, the electron energy gain was observed to be limited by phase detuning between the accelerated electrons and the plasma wave.

A fundamental limitation in all plasma-based accelerators is electron-phase detuning, i.e., accelerated electrons (with $v \rightarrow c$ ) outrun the plasma wave (with $v_{p} \simeq v_{g}<c$ ). Acceleration will cease once the electrons phase advance a distance $\left(v-v_{p}\right) t \simeq \lambda_{p} / 2$ relative to the plasma wave, where $\lambda_{p}=2 \pi c / \omega_{p}$ is the plasma wave length. In the laboratory frame, this corresponds to a detuning distance of $L_{t} \simeq \gamma_{p}^{2} \lambda_{p}$, where $v=c$ has been assumed. It can be shown ${ }^{13}$ that the maximum energy gain, $\Delta W_{\max }$, of a trapped electron in a 1-D plasma wave of amplitude $E_{z}$ is $\Delta W_{\max } \simeq 4 m_{e} c^{2} \gamma_{p}^{2} E_{z} / E_{0}$ for $E_{z}^{2} / E_{0}^{2} \ll 1$, and in the nonlinear limit, $\Delta W_{\max } \simeq 2 m_{e} c^{2} \gamma_{p}^{2}\left(E_{z} / E_{0}\right)^{2}$ for $E_{z}^{2} / E_{0}^{2} \gg 1$. For example, for a fixed value of $\varepsilon=E_{z} / E_{W B}=0.25$ and a laser wavelength of $\lambda=1 \mu \mathrm{m}, \Delta W_{\max } \simeq 3.4 \mathrm{GeV}$ for $n_{e 0}=10^{18} \mathrm{~cm}^{-3}\left(E_{W B}=7.3 \mathrm{GV} / \mathrm{cm}\right)$, whereas $\Delta W_{\max } \simeq 3.4 \mathrm{TeV}$ for $n_{e 0}=10^{16} \mathrm{~cm}^{-3}$ $\left(E_{W B}=2.4 \mathrm{GV} / \mathrm{cm}\right)$, where $\gamma_{p} \simeq \omega / \omega_{p} \gg 1$ has been assumed. Notice that for a fixed $\varepsilon$, $\Delta W_{\max } \simeq 4 m_{e} c^{2} \gamma_{p}^{3} \varepsilon^{2} \sim n_{e 0}^{-3 / 2}$, assuming $E_{z}^{2} / E_{0}^{2} \gg 1$ and $\gamma_{p}^{2} \gg 1$. Hence, at the high densi- 
ties required either for self-modulation or for the use of an ultrashort pulse in the standard LWFA, $\gamma_{g}$ is relatively low and acceleration is limited by electron phase detuning.

In a previous paper ${ }^{14}$, we proposed an alternative accelerator concept, which we call the resonant laser-plasma accelerator (RLPA), that combines the virtues of these others, but has the following additional advantages: (i) by utilizing a train of laser pulses with independently adjustable pulse widths and interpulse spacings, which are varied in an optimized manner, resonance with both the changing plasma-wave period and phase resonance width can be maintained in the nonlinear regime, and the maximum plasma-wave amplitude is achieved; (ii) lower plasma densities can be used, thus avoiding electron-phase detuning; and (iii) lower peak laser intensities can be used, thus allowing for a reduction of laserplasma instabilities. In this paper we investigate the RLPA concept ${ }^{14-16}$ in greater detail, including important issues such as (1) the sensitivities of the wake field to changes in the laser and plasma parameters, and (2) nonlinear Landau damping of the wave.

This paper is concerned with determining the characteristics of the plasma wave generated by a nonevolving, optimized laser pulse train in 1D. The laser intensity profile of the pulse train is assumed to be nonevolving and a function of only the variable $\zeta=z-v_{p} t$, where $z$ is the axial propagation distance and $v_{p}=v_{g}$ is assumed. Neglected are various effects which could effect the evolution of the laser pulse train, such as diffraction or pump depletion. In the absence of some form of optical guiding, a laser pulse will diffract after propagating a distance characterized by the Rayleigh length, $Z_{R}=\pi r_{0}^{2} / \lambda$, where $r_{0}$ is the minimum laser spotsize at focus. For a tightly focused laser pulse, $Z_{R}$ can be relatively short. However, it has been observed both numerically ${ }^{9}$ and experimentally ${ }^{17}$ that a preformed plasma channel can be used to guide the laser pulse and prevent diffraction. Pump depletion refers to the process by which the laser pulse loses energy as it generates a plasma wave. A rough estimate for the pump depletion length, $L_{d}$, is given by equating the energy left behind in the plasma wave to the initial energy in the laser pulse train, i.e., $L_{d} E_{z}^{2} \simeq c \tau_{t o t} E_{L}^{2}$. Here, it is assumed that the laser pulse train consists of pulses of with equal intensities $\left(E_{L}\right.$ is the amplitude of the electric field of the laser pulse) and the sum of the pulse durations is $\tau_{\text {tot }}$. Consider laser pulses with an intensity $I \simeq 10^{18} \mathrm{~W} / \mathrm{cm}^{2}\left(E_{L} \simeq 30 \mathrm{GV} / \mathrm{cm}\right.$ for $\left.\lambda=1 \mu \mathrm{m}\right)$ and a total duration of $\tau_{\text {tot }}=1 \mathrm{ps}$. For a plasma wave amplitude of $E_{z} \simeq 0.5 \mathrm{GV} / \mathrm{cm}, L_{d} \simeq 3$ $\mathrm{cm}$. For propagation distances $\geq L_{d}$, pump depletion effects must be considered. The use of an active medium has been suggested as a method to overcome pump depletion ${ }^{18}$.

In Sec. 2, analytic solutions and sample calculations for a square pulse train are presented to demonstrate the advantage of the use of multiple pulses in terms of amplitude and energy efficiency. In Sec. 3, numerical results are presented of pulse trains of both the square and sine shapes. Comparison of the different acceleration schemes suggests that the RLPA is more efficient than either the PBWA or the LWFA. We also discuss the sensitivities of the various plasma wave generation schemes to changes in the laser and plasma parameters, such as laser intensity (Sec. 3.2.6), laser pulse widths and interpulse spacings (Sec. 3.2.4), and plasma density (Sec. 3.2.5). Kinetic effects investigated by use of a PIC code simulation are discussed in Sec. 4. Pulse-shaping techniques are discussed in Sec. 5. Conclusions are presented in Sec. 6. 


\section{ANALYSIS}

The laser-plasma interaction is modeled by the relativistic fluid-Maxwell equations. The laser pulse is described by the normalized transverse vector potential, $\vec{a}=e \vec{A}_{\perp} / m_{e} c^{2}$. The laser envelope, $|a|$, is assumed to be nonevolving and a function of only $\zeta=z-v_{g} t$, where $v_{g}$ is the group velocity (assumed constant). Circular polarization is assumed, i.e., $a^{2}=$ $a^{2}(\zeta)$. The quantity $a^{2}$ is related to the laser wavelength $\lambda$ and intensity $I$ by $a \simeq 6 \times$ $10^{-10} \lambda[\mu \mathrm{m}] I^{1 / 2}\left[\mathrm{~W} / \mathrm{cm}^{2}\right]$. The plasma response is described by the normalized electrostatic potential, $\phi=e \Phi / m_{e} c^{2}$, which in the 1-D limit obeys the nonlinear Poisson equation ${ }^{14-16,20}$

$$
\frac{d^{2}}{d \zeta^{2}} \phi=k_{p}^{2} \gamma_{g}^{2}\left[\beta_{g}\left(1-\frac{\left(1+a^{2}\right)}{\gamma_{g}^{2}(1+\phi)^{2}}\right)^{-1 / 2}-1\right],
$$

where $\beta_{g}=v_{g} / c, \gamma_{g}=\left(1-\beta_{g}^{2}\right)^{-1 / 2}$ and $k_{p}=\omega_{p} / c$ is the plasma wave number. In deriving Eq. (1), $\phi$ was assumed to be a function of only $\zeta$, i.e., $v_{p} \simeq v_{g}$. In the limit $a^{2} \ll 1, \gamma_{g}=$ $\omega / \omega_{p}$ (nonlinear corrections are discussed $i^{21}$ ). As previously mentioned, the laser pulse structure is assumed to be nonevolving. This ignores various effects, such as diffraction, pump depletion and laser-plasma instabilities.

\subsection{Square Pulses}

Several properties of the plasma wave can be determined analytically from Eq. (1) for a series of square laser pulses. When $a^{2}$ is constant, Eq. (1) can be integrated to yield

$$
x^{\prime 2}=2 \gamma_{g}^{2}\left\{\left(x_{0}-x\right)+\beta_{g}\left[\left(x^{2}-\gamma_{\perp}^{2} / \gamma_{g}^{2}\right)^{1 / 2}-\left(x_{0}^{2}-\gamma_{\perp}^{2} / \gamma_{g}^{2}\right)^{1 / 2}\right]\right\}
$$

where $x=1+\phi, \gamma_{\perp}=\left(1+a^{2}\right)^{1 / 2}$ and $x_{0}$ is an initial condition, i.e., $x=x_{0}$ at $x^{\prime}=0$. Here, $x^{\prime}=k_{p}^{-1} d \phi / d \zeta$ and is the normalized axial electric field of the plasma wave, i.e., $x^{\prime}=\hat{E}_{z} \equiv E_{z} / E_{0}$, where $E_{0}=m_{e} c^{2} k_{p} / e$ (sometimes referred to as the cold, nonrelativistic wavebreaking field ${ }^{6}$ ).

Consider an optimized square pulse train where $a_{n}$ is the amplitude of the $n^{\text {th }}$ pulse. For the first pulse, Eq. (2) is solved with $a=a_{1}$ and the initial condition $x_{0}=x_{\text {min }_{0}}=1$. Equation (2) is integrated from the front of the pulse to the back. The optimal pulse length, $L_{1}$, is determined by the $\zeta$ distance required to reach maximum potential within the pulse, i.e., $x^{\prime}=0$ and $x=x_{\max _{1}}$. The wake behind the first pulse is given by solving Eq. (2) with $a^{2}=$ 0 using the initial conditions $x^{\prime}=0$ and $x_{0}=x_{\max _{1}}$. The potential of the wake oscillates between $x_{\max _{1}}$ and $x_{\min _{1}}$. The distance required to reach the minimum potential, $x^{\prime}=0$ and $x=x_{\min _{1}}$, is defined to be one half the nonlinear plasma wavelength, $\lambda_{N_{1}} / 2$. The optimal spacing between the first and second pulse is determined by placing the front of the second pulse at the position in the wake of the first pulse for which $x^{\prime}=0$ and $x=x_{\min _{1}}$. Hence, the optimal spacing between the first and second pulse is some odd multiple of $\lambda_{N_{1}} / 2$. In general, for an optimized square pulse train, it can be shown that the amplitude of the wake behind the $n^{\text {th }}$ pulse oscillates between $x_{\min _{n}} \leq x \leq x_{\text {max }_{n}}$, where

$$
x_{\min _{n}}=\gamma_{g}^{2}\left[x_{\max _{n}}\left(1+\beta_{g}^{2}\right)-2 \beta_{g}\left(x_{\max _{n}}^{2} / \gamma_{g}^{2}\right)^{1 / 2}\right]
$$




$$
x_{\max _{n}}=\gamma_{g}^{2}\left[x_{\min _{n-1}}\left(1+\beta_{g}^{2}\right)-2 \beta_{g}\left(x_{\min _{n-1}}^{2}-\gamma_{\perp_{n}}^{2} / \gamma_{g}^{2}\right)^{1 / 2}\right] \text {. }
$$

Here, $\gamma_{\perp_{n}}=\left(1+a_{n}^{2}\right)^{1 / 2}$ and $x_{\min _{0}} \equiv 1$. Furthermore, the maximum electric field amplitude behind the $n^{\text {th }}$ pulse is given by

$$
\hat{E}_{\max _{n}}^{2}=2 \gamma_{g}^{2}\left[x_{\max }-1 / \gamma_{g}^{2}-\beta_{g}\left(x_{\max _{n}}^{2}-1 / \gamma_{g}^{2}\right)^{1 / 2}\right]
$$

where $\hat{E}_{\text {max }_{n}}=E_{\max } / E_{0}$. In deriving Eqs. (3)-(5), the spacing between pulses and the pulse lengths are assumed to be optimized, such that the $n^{t h}$ pulse begins at $x=x_{m i n}$ and ends at $x=x_{\max _{n}}$. Both the optimal width $L_{n}$ of the $n^{\text {th }}$ pulse and the nonlinear wavelength of the wake behind the $n^{\text {th }}$ pulse (and, hence, the optimal spacing between pulses) increase with increasing $n$. Wave breaking occurs when the electron fluid velocity becomes equal to the plasma wave phase velocity $v_{g}$. When this occurs, the electron fluid density becomes singular. From Eq. (1), wave breaking occurs when $x_{\min _{n}} \rightarrow 1 / \gamma_{g}$, which implies $x_{\max } \rightarrow$ $x_{W B}=\left(2 \gamma_{g}^{2}-1\right) / \gamma_{g}$. This corresponds to a wave-breaking electric field ${ }^{5}$ of $\hat{E}_{W B}^{2}=2\left(\gamma_{g}-1\right)$, or $E_{z}=E_{W B}$.

Numerical solutions to Eq. (1) indicate that for $x^{2} \ll x_{W B}^{2}$, Eq. (1) can be approximated by the limit $\beta_{g} \rightarrow 1$, i.e.,

$$
2 x^{\prime \prime}=\gamma_{\perp}^{2} / x^{2}-1
$$

where the prime denotes $k_{p}^{-1} d / d \zeta$. For a series of optimized square pulses, analytic solutions can also be readily obtained from this reduced equation. In particular,

$$
\begin{aligned}
& x_{\max _{n}}=\gamma_{\perp_{1}}^{2} \gamma_{\perp_{2}}^{2} \cdots \gamma_{\perp_{n}}^{2}, \\
& \hat{E}_{\text {max }_{n}}=x_{\text {max }}^{1 / 2}-x_{\max _{n}}^{-1 / 2},
\end{aligned}
$$

and $x_{\min _{n}}=1 / x_{\max _{n}}$. Furthermore, the optimal width of the $n^{\text {th }}$ pulse, $L_{n}$, and the nonlinear wavelength of the wake behind the $n^{t h}$ pulse, $\lambda_{N_{n}}$, are given by

$$
\begin{gathered}
L_{n}=\left(2 / k_{p}\right) x_{\max _{n}}^{1 / 2} E_{2}\left(\rho_{n}\right), \\
\lambda_{N_{n}}=\left(4 / k_{p}\right) x_{\max _{n}}^{1 / 2} E_{2}\left(\hat{\rho}_{n}\right),
\end{gathered}
$$

where $E_{2}$ is the complete elliptic integral of the second kind, $\rho_{n}^{2}=1-\gamma_{\perp_{n}}^{2} x_{\max _{n}}^{-2}$ and $\hat{\rho}_{n}^{2}=$ $1-x_{\max _{n}}^{-2}$. The optimal spacing between the end of the $n^{t h}$ pulse and the $n^{t h}+1$ pulse is an integer multiple of $\lambda_{N_{n}}$. Note for equal pulse amplitudes, i.e., $a_{1}=a_{2}=\ldots \equiv a_{0}, x_{\max }=$ $\gamma_{\perp_{0}}^{2 n}=\left(1+a_{0}^{2}\right)^{n}$. In the limit $x_{\text {max }_{n}}^{2} \gg 1, k_{p} L_{n} \simeq 2 \gamma_{\perp_{0}}^{n}$, and $k_{p} \lambda_{N n} \simeq 4 \gamma_{\perp_{0}}^{n}$, and $x_{\max _{n}}^{\prime} \simeq k_{p} \gamma_{\perp_{0}}^{n}$. Several recent papers have addressed various aspects of this problem ${ }^{14-16}$.

The maximum normalized electric field, $\hat{E}_{\max }=x_{\max }^{\prime}=E_{\max } / E_{0}$, for an optimized train of $n$ square pulses of equal amplitudes, is plotted in Fig. 1 versus the quantity $a_{T}^{2} \simeq n a_{0}^{2}$, using the above analytical results. For $\gamma_{g} \gg 1$ and $x^{2} \ll x_{W B}^{2}, \hat{E}_{\max }$ is approximately independent of $n_{e 0}$. The curves show the result for $1,3,5,10$, and 100 pulses. Figure 1 indicates that just a few optimized square pulses are far more efficient than a single pulse. For example, at $n_{e 0}=10^{15} \mathrm{~cm}^{-3}\left(\lambda=1 \mu \mathrm{m}, \gamma_{g} \simeq 10^{3}, E_{W B} \simeq 1.4 \mathrm{GV} / \mathrm{cm}\right)$, three square pulses can be used with an intensity $I=2.7 \times 10^{18} \mathrm{~W} / \mathrm{cm}^{2} /$ pulse $\left(a_{0}^{2}=1\right)$ and a total pulse train energy of 
$I \tau_{t o t}=20 \mathrm{MJ} / \mathrm{cm}^{2}$ to produce $E_{z}=0.1 \mathrm{GV} / \mathrm{cm}$. Here, $\tau_{t o t}$ is the sum of the pulse durations in the train and $2.7 a_{0}^{2} \simeq 10^{-18} \lambda^{2}[\mu \mathrm{m}] I\left[\mathrm{~W} / \mathrm{cm}^{2}\right]$. A single pulse at $n_{e 0}=10^{15} \mathrm{~cm}^{-3}$ requires $I=3 \times 10^{19} \mathrm{~W} / \mathrm{cm}^{2}\left(a_{0}^{2}=11\right)$, over an order of magnitude higher intensity than in each pulse in the train, and a total energy six times greater $\left(I \tau_{t o t}=120 \mathrm{MJ} / \mathrm{cm}^{2}\right)$, to produce this same $E_{z}$. (A low density was chosen for this example so that finite rise-time effects could be neglected, as discussed Sec. 3.2.1.) Figure 1 indicates that the amplitude-efficiency advantage of multiple pulses increases with increasing number of pulses $n$ or total laser intensity $a_{0}^{2}$. Figure 2 shows the ratio of the maximum field achieved with a train of pulses $\left(\hat{E}_{\max }\right)$ over that achieved with an equivalent-energy single pulse $\left(\hat{E}_{\max }\right)$ versus $a_{n}^{2}$, demonstrating the energy-efficiency of the RLPA as compared with the LWFA.

\section{NUMERICAL OPTIMIZATION}

\subsection{Square pulses}

Figure 3 shows an example of an optimized square pulse train $\left(n=4, a_{0}=1.2, n_{e 0}=10^{16}\right.$ $\mathrm{cm}^{-3}$ ), as obtained by a numerical solution of Eq. 1 , in which the widths and spacing between pulses are varied in order to maximize $x_{\max }$. For numerical reasons, we used non-ideal square pulses that have small but finite rise times. As will explained in Sec. 3.2.1, only in the limit of low density, as was used in the example of Fig. 3, do numerically optimized trains of pulses with finite rise times reproduce the results analytically predicted in the ideal case (Sec. 2.1). The laser pulses are optimally located in the regions where $d \phi / d \zeta>0$. If the laser pulse is located in the region of $d \phi / d \zeta<0$, it will absorb energy from, and reduce the amplitude of, the plasma wave. Likewise, if it is in the region of $d \phi / d \zeta>0$, it will impart energy to, and increase the amplitude of, the plasma wave. The sign of $d \phi / d \zeta$ determines whether the group of background electrons in the frame of reference moving at speed close to $c$ will have positive or negative velocity relative to the frame of reference. The electrons with negative (positive) relative velocity will be accelerated (decelerated) by the laser pulse, downshifting (upshifting) the laser frequency, i.e., this determines the direction of energy of transfer. When a train that is not optimized is used, for instance fixed interpulse spacings (as in the case of the PBWA), $x_{\max }$ reaches some saturated value before being driven down by destructive interference when the pulses become out of phase with the wave, i.e., when they are located in regions where $d \phi / d \zeta<0$. This is referred to as resonance detuning. The plasma wave is driven most effectively near $\phi=\phi_{\min }$ (because that is where $n_{e}$ is maximum), and least effectively as $\phi \rightarrow \phi_{\max }$.

\subsection{Sine pulses}

The above results are valid in the limits of either infinitesimally short rise times, or low density. In practice, the rise time $\tau_{\text {rise }}$ of a pulse directly out of a laser is finite and determined by the bandwidth of the laser amplifiers; e.g., currently, the minimum amplified pulse width is $\tau_{\min } \simeq 50 \mathrm{fs}^{19}$. In order to study the effects of plasma density and finite rise times on efficiency, we now consider pulses with an envelope profile $a(\zeta)$ given by a half-period of a sine function. (That Gaussian profiles give qualitatively similar results is verified in other 
simulations.)

In Fig. 4(a), we plotted the wake field resulting from single pulse excitation (LWFA) including fast oscillations of the laser pulse. For this example, $n_{e 0}=10^{16} \mathrm{~cm}^{-3}$ and $a_{0}=2$. The high-frequency density fluctuation inside the laser pulse envelope is due to electron quiver motion by the high-frequency oscillating electric field of the pulse. Figure 4(b) shows an example of a sine pulse train $\left(n=4, a_{0}=1.2, n_{e 0}=10^{16} \mathrm{~cm}^{-3}\right)$ that was optimized numerically. The first pulse in Fig. 4(b) has an optimum pulse width $\tau=\tau_{\text {opt }}=940$ fs (resonant with $n_{e 0}=10^{16} \mathrm{~cm}^{-3}$ and $a_{0}=1.2$ ) and the final pulse has $\tau=\tau_{o p t}=\tau_{\text {min }} \simeq 200$ fs $\left(I \tau_{\text {tot }}=2.1 \mathrm{MJ} / \mathrm{cm}^{2}\right)$, which gives $E_{z}=0.18 \mathrm{GV} / \mathrm{cm}(\varepsilon=0.07)$. As in the square wave case, $\lambda_{N_{n}}$, and thus the spacing between pulses, increases with each succeeding pulse as $x_{\max }$ increases.

\subsubsection{Plasma wave phase resonance region}

Note that whereas $\tau_{o p t} \sim \lambda_{N_{n}} / c$ for succeeding square wave pulses increases with increasing $x_{\max }$, the opposite is true for multiple sine pulses. This difference arises because, whereas for square pulses $\tau$ is independent of $\tau_{\text {rise }}$, for sine pulses $\tau \simeq 2 \tau_{\text {rise }}$. It is more advantageous to have a short sine pulse width $\left(\tau \ll \lambda_{N_{n}} / c\right)$, so that the highest pulse amplitude is reached near $\phi_{\min }$ (where it is most effective in driving the plasma wave), than to have a long sine pulse width ( $\tau \simeq \lambda_{N_{n}} / c$ ), so that the pulse is driving the wave for a longer time, albeit mostly when it is less effective (away from $\phi_{\min }$ ). Sine pulses are found to be more effective than square pulses for this same reason. For the later sine pulses, $\tau_{o p t}$ is found to be approximately given by the width of the region between where $\phi<0$ and $d \phi / d \zeta>0$, which defines a "phase resonance width" $L_{r e s}$. The physical origin of $L_{r e s}$ is that in this region (i) the ponderomotive force of the laser pulse is in the right phase with the electron motion to give energy to the plasma wave, and (ii) the density of electrons with which the light pulse can interact is highest.

For the wake behind the $n^{t h}$ pulse, $L_{\text {res }}$ can be determined from Eq. (1) in the limit $v_{g}=c$,

$$
L_{r e s}=\left(2 / k_{p}\right) x_{\max }^{1 / 2}\left|E_{2}\left(\pi / 2, \hat{\rho}_{n}\right)-E_{2}\left(\alpha_{1}, \hat{\rho}_{n}\right)\right|,
$$

where $\hat{\rho}_{n}^{2} \sin ^{2} \alpha_{1}=1-x_{\max _{n}}^{-1}$. In the limit $x_{\max _{n}}>>1, L_{\text {res }} \rightarrow k_{p}^{-1} x_{\max _{n}}^{-1 / 2} \simeq 1 / \hat{E}_{\max _{n}}$ and, hence, the resonance becomes sharper $\left(Q \equiv \lambda_{N n} / L_{r e s} \sim x_{\text {max }_{n}}\right)$.

Figure 6 shows a plot of $L_{r e s} / c$, which approximates $\tau_{o p t}$, versus $\varepsilon=E_{z} / E_{W B}$ for various densities. Notice that, in the regime of high $n_{e 0}$, finite rise-time effects become important at high $\varepsilon$, i.e., $\tau_{\text {opt }}$ decreases below $\tau_{\min }$ as $\varepsilon$ increases beyond a critical value (e.g., $L_{\text {res }} / c<50$ fs for $\varepsilon>0.14$, at $n_{e 0}=10^{16} \mathrm{~cm}^{-3}$ ). Since pulses with $\tau<\tau_{\min }$ cannot currently be produced, the later pulses in a train will not be optimized. Although the later pulses with $\tau=\tau_{\min }>\tau_{o p t}$ will continue to increase $x_{\max }$, they will do this less effectively than a train in which all pulses are of optimal widths. Consequently, although for these later pulses the sensitivity to changes in $\lambda_{N}$ is reduced (as will be shown in Sec. 3.2.4), a pulse train in this high- $n_{e 0}$ regime can be less amplitude efficient than a single optimized pulse at the same density; i.e., a greater $I \tau_{t o t}$ is required for the pulse train to achieve a given $E_{z}$ at fixed $n_{e 0}$. High $n_{e 0}$, however, is unfavorable for electron acceleration because of electron-phase detuning, $\Delta W_{\text {max }} \sim E_{z}^{2} n_{e 0}^{-3 / 2}$, as will be discussed in greater detail in the next subsection. 


\subsubsection{Efficiency comparison between RLPA and LWFA}

Figure 6 indicates that, for low $n_{e 0}$ and up to the previously mentioned critical value of $\varepsilon$, the condition $\tau_{o p t} \simeq L_{r e s} / c \geq \tau_{\min } \simeq 50$ fs can be satisfied for all of the pulses in a train [as was the case of Fig. 4(b)]. Consequently, multiple sine pulses in this regime are found to be similar to ideal square pulses in that a pulse train is more amplitude efficient than a single pulse at the same density. Specifically, 8 times higher intensity ${ }^{22}\left(a_{0}=3.4\right.$, or $I=3.1 \times 10^{19}$ $\left.\mathrm{W} / \mathrm{cm}^{2}\right)$, corresponding to 2.6 times more energy $\left(I \tau_{t o t}=5.4 \mathrm{MJ} / \mathrm{cm}^{2}\right)$, is required of a single pulse $\left(\tau=\tau_{o p t}=700 \mathrm{fs}\right.$ for $\left.n_{e 0}=10^{16} \mathrm{~cm}^{-3}\right)$ to reach the same value of $E_{z}(0.18 \mathrm{GV} / \mathrm{cm})$ as is reached by the train of Fig. 4(b). In terms of energy efficiency, a single pulse with the same intensity and pulse width as the first pulse in Fig. 4(b), corresponding to 0.42 times the laser energy $\left(I \tau_{t o t}=0.91 \mathrm{MJ} / \mathrm{cm}^{2}\right)$, results in a 3.9 -times-smaller $E_{z}(46 \mathrm{MV} / \mathrm{cm})$. Reducing the intensity required to reach large plasma-wave amplitudes also reduces strongly driven instabilities, such as stimulated Raman scattering, self-focusing, or filamentation, which disrupt either the plasma wave or the laser beam. Pulse-to-pulse phase incoherence of the high-frequency laser oscillations can also reduce instabilities.

In order to drive the same $E_{z}$ with the same $I$ as a sine pulse train, a higher $n_{e 0}$ must be used with a single sine pulse. (Recall that, for a single pulse, $E_{z} \sim n_{e 0}^{1 / 2} I$ for $a_{0}^{2}<1$.) Thus, the same value of $E_{z}=0.18 \mathrm{GV} / \mathrm{cm}$ as is reached by the train in Fig. 4(b) is obtained by an equivalent-intensity single pulse $\left(a_{0}=0.7\right)$ with $\tau=\tau_{o p t}=90 \mathrm{fs}$ at $n_{e 0}=10^{18} \mathrm{~cm}^{-3}$, and with 70 times less energy $\left(I \tau_{t o t}=30 \mathrm{~kJ} / \mathrm{cm}^{2}\right)$. But, because energy gain favors low $n_{e 0}$, the pulse train in Fig. 4(b) is 1000 times more "acceleration efficient"; i.e., $\Delta W_{\max }=13.7$ $\mathrm{TeV}$ for the pulse train, whereas $\Delta W_{\max }=13.7 \mathrm{GeV}$ for the single pulse. Thus, a pulse train of equivalent intensity -at either equal or lower $n_{e}$ - is more acceleration efficient than a single pulse ${ }^{23}$.

\subsubsection{Efficiency comparison between RLPA and PBWA}

Thus far, the RLPA concept has been compared only to the LWFA; in this section, it is compared to the PBWA. In Fig. 5(a), the unperturbed plasma wave frequency was used for the beat frequency in a PBWA pulse train, $\Delta \omega \sim \omega_{p}$. However, as expected in this nonlinear regime, resonance detuning between the plasma wave and the PBWA laser train is observed. Therefore, for a more reasonable comparison, the pulse width for the PBWA needs to be optimized for a given plasma density, as was done for the RLPA, but in this case with the constraint that the pulse widths, pulse amplitudes, and interpulse spacings are kept constant for all pulses in the train. The PBWA optimized in this manner is shown in Fig. 5(b). A beatwave wavelength greater than the one corresponding to the unperturbed density $\lambda_{p}$ is found to be optimum ${ }^{24}$, compensating for the increase in the nonlinear wavelength $\lambda_{N}$ with increasing beatwave amplitude. As can be seen from Fig. 5(b), the net effect is to move the peak of the pulses closer to $L_{\text {res }}$ and avoid resonance detuning. Although the final wake of the optimized PBWA is found in the example of Fig. 5(b) to be similar to that in the RLPA scheme for comparable laser pulse intensities, it should be emphasized that much more energy was required for the former.

It is useful to compare the wake fields produced by the various concepts given equal total laser energies, since that is the technological limitation imposed by the type of lasers capable 
of the high-intensities required ${ }^{25}$. The intensity and pulse width were varied in such a way that the total laser energy and number of pulses were kept the same for both the PBWA and the RLPA. It is found that the optimized PBWA is less energy-efficient than either the RLPA or the LWFA. For example, a PBWA pulse train with $a_{0}=1.07, \tau=1060 \mathrm{fs}$, where $\tau$ is the pulse width for each pulse, and total energy in the pulse train equal to $I \tau_{t o t}=3.29 / \mathrm{cm}^{2}$, produced a normalized wake-field amplitude of $E_{z} / E_{0}=0.377$. An equivalent-energy RLPA train $\left(a_{0}=1.6, \tau_{t o t}=1900 \mathrm{fs}\right)$ gave $E_{z} / E_{0}=2.98$, which is 7.9 times larger. In another example, a LWFA single pulse with $I \tau_{t o t}=5.05 \mathrm{MJ} / \mathrm{cm}^{2}\left(a_{0}=3.27, \tau=700 \mathrm{fs}\right)$ produced a wake larger by a factor of $1.23, E_{z} / E_{0}=1.69$, than an equivalent-energy PBWA with $a_{0}=1.2$ and $\tau=1300 \mathrm{fs}$, which generated $E_{z} / E_{0}=1.38$. Thus, based on the previous discussion, the RLPA is the most energy efficient of all three schemes.

\subsubsection{Wake-field amplitude vs interpulse spacing and pulse width}

The sensitivity of the growth of $\hat{E}_{\max }$ to changes in the pulse widths $\tau$ and interpulse spacings $\lambda_{N_{n}}$ of the laser pulses of Fig. 4(b) was studied numerically. It is governed by both the number of pulses and the $Q$ of the resonance, where $Q \sim x_{\max }$ is as defined in Sec. 3.2.1. This can be seen from Fig. 7 in which we plot the maximum electric field $\hat{E}_{\text {max }}$ produced by varying both $\tau$ and $\lambda_{N_{n}}$, for the second $n=2$ (a), third $n=3$ (b), and fourth $n=4$ (c) pulses of the train shown in Fig. 4(b). For instance, from Fig. 7(c), it appears that the fourth pulse $n=4$ is highly sensitive to absolute changes in $\tau$ or $\lambda_{N_{n}}$ in the vicinity of $\tau=\tau_{o p t}$.

It can clearly be seen from Fig. 7(c) that the wake from pulses with $\tau>\tau_{\text {opt }}$ are found to be less sensitive to changes in interpulse spacing than those with $\tau=\tau_{o p t}$, without sacrificing much efficiency. For instance, if the pulse width of the last pulse $(n=4)$ were $\tau=300 \mathrm{fs}$ $\simeq 1.5 \tau_{\text {opt }}$ (instead of $\tau_{\text {opt }}$ ), it is found that a decrease in the optimal spacing between the last and the third pulse $\left(\lambda_{N_{3}}\right)$ by $25 \mathrm{fs}$ (corresponding to $\delta \lambda_{N_{3}} / c \tau_{o p t}=13 \%$ ) results in a decrease of $E_{z}$ (from the value obtained using $\tau=\tau_{o p t}$ and the optimal position) by only $2.2 \%$ (instead of $5 \%$ ). Note, in the $\tau=1.5 \tau_{o p t}$ case, $I \tau_{t o t}=2.2 \mathrm{MJ} / \mathrm{cm}^{2}$, corresponding to a laser pulse train energy increase of only $4.5 \%$.

The added pulses can also absorb the plasma wave, i.e., the maximum electric field $\left(\hat{E}_{\text {max }_{n}}\right)$ can be reduced to a value below that without it $\left(\hat{E}_{m a x_{n-1}}\right)$, when the spacing is reduced such that the pulse becomes located in the $d \phi / d \zeta<0$ region. Absorption can be optimized just as amplification can, by varying $\tau$ and $\lambda_{N}$, with the maximum amount of absorption equaling the maximum amount of amplification. The second pulse can in fact totally absorb the plasma wave produced by the first pulse ${ }^{15}$, the energy of the plasma wave going into upshifting the frequency of the light ${ }^{26}$. The amplitude is less sensitive to an increase in the spacing, since this moves the pulse further from the $d \phi / d \zeta<0$ region, and thus the wake continues to be enhanced, but less effectively. As $\lambda_{N}$ increases beyond its optimum value, $\hat{E}_{\max }$ asymptotes to the value it had without the pulse, $\hat{E}_{\max _{n-1}}$. Thus, the larger the value of $n$, the less the sensitivity to spacing, since the value of $\hat{E}_{m a x}$ is large to begin with, and thus the relative change, $\Delta \hat{E}_{\max _{n}} / \hat{E}_{\text {max }_{n-1}}$ cannot be as large as it is for, say, the $n=2$ pulse, for which $\hat{E}_{\text {max }_{n-1}}$ is smaller. (See the scaling change of $\hat{E}_{\max }$ for the three plots of Fig. 7.) 


\subsubsection{Wake-field amplitude vs plasma density}

Since the exact resonant plasma density is difficult to produce with current technology, we must consider how stable the final RLPA wake field is to variation of the plasma density. In Fig. 8(a), the sensitivity of the wake field versus the plasma density for the pulse train in Fig. 4(b) is shown. The density resonance width is 0.51 , which is defined as $\Delta n / n_{0}=$ $\left(n_{U}-n_{L}\right) / n_{0}$, where $n_{U}$ and $n_{L}$ are the upper and lower values of the density for which the wake amplitude is half of its peak value (which it has at the resonant density $n_{0}$ ). For comparison, the density resonance for the PBWA pulse train of Fig. 5(b) is shown in Fig. 8 (b), with a corresponding density resonance width found to be equal to 0.62 . The arrow indicates the density corresponding to the resonant density in the linear approximation, $\Delta \omega=\omega_{p}\left(n_{e}\right)$. Thus despite the much greater efficiency of the RLPA than the PBWA, their sensitivities to density variation are similar. Achieving a density uniformity meeting this requirement should pose no significant technological challenges - at least for a proof-ofprinciple experiment-since, in fact, by use of multiphoton ionization ${ }^{27}$, it has already been greatly exceeded in the laboratory over a distance $[O(10 \mathrm{~cm})]$ that is greater than the short distance discussed in Sec. $3.2\left[4 \lambda_{N} \sim O(\mathrm{~mm})\right]$.

\subsubsection{Wake-field amplitude vs laser intensity}

In addition to density variation, shot-to-shot laser intensity fluctuations can result in detuning. Figure 9(a) shows the dependence of wake field amplitude on the laser intensity for the RLPA. As usual we assume here that the intensities of all pulses in the train are the same. Note the multiple peaks and sudden discontinuities in the slope of the curve. They correspond to the various pulses coming in and out of resonance as $\hat{E}_{\max }$ and thus $\lambda_{N}$ change with increasing intensity. The peak at $a_{0}^{2}=1.44$ corresponds to optimization of all pulses. As the intensity $\left(a_{0}^{2}\right)$ increases, the position of the fourth pulse moves toward the absorption region $(d \phi / d \zeta<0)$ and thus $x_{\max }$ becomes reduced. At $a_{0}^{2}=1.6$, the fourth pulse moves into the emission region again $(d \phi / d \zeta>0)$ and there is a sharp discontinuity. Another discontinuity appears at $a_{0}^{2}=2.1$ as the third pulse moves from the absorption to the emission region. The peak at $a_{0}^{2} \sim 2.3$ corresponds to the fourth pulse reaching resonance again. Figure 9(b), which shows the sensitivity of the PBWA, is a smooth curve with only one peak since, unlike the RLPA, the PBWA pulses are much longer than $L_{r e s}$, and the optimum intensity for the PBWA in this example is the highest intensity plotted $\left(a_{0}^{2}=2.5\right)$. However, as can be seen from Fig. 9(a), the amplitude fluctuations of the RLPA are in the worst case only $20 \%$ for a $10 \%$ change in laser intensity, which does not represent a serious problem since shot-to-shot intensity stabilities of $\leq 5 \%$ are achievable.

\section{PARTICLE-IN-CELL CODE SIMULATIONS}

In order to study kinetic effects, we used a particle-in-cell (PIC) code with one spatial dimension and three velocity dimensions. The simulation is fully relativistic and incorporates all of Maxwell's equations. In order to simulate a laser pulse, one boundary becomes an antenna. A sine wave oscillates at the laser's wavelength, in this case $1 \mu \mathrm{m}$. To get the correct pulse shape, another sine wave modulates the laser wave so that the pulse has the shape of 
a half sine-wave equal to the pulse width. A spatial grid was set up so that one laser wavelength was equal to 20 grid points. For a particle density $n_{p}$ of $10^{16} \mathrm{~cm}^{-3}$, as chosen in the previous fluid model simulations, the plasma wavelength $\lambda_{p}$ is $279 \mu \mathrm{m}$, or $940 \mathrm{fs}$. The total domain of the simulation is $10 \lambda_{p}$ in length. The electron-ion mass ratio is $1 / 1836$, with the electron charge chosen to give the correct $\lambda_{p}$ for the above $n_{p}$ with 10 particles/cell. The first simulation run, shown in Fig. 11(a), was for LWFA with a pulse width equal to $\lambda_{p}$. Qualitatively it has the same characteristics as that of the fluid model, although the wavelength of the resultant wake field differs by about $10 \%$. Comparison of these two results validates the quasi-static approximation and the assumption of nonevolving pulse-shapes used in the fluid code, but, of course, only for the short distances studied.

Because of the computing expense involved in running the PIC code, optimization of the RLPA could not be done by variation of parameters as was done with the fluid code. A good approximation, however, was made by performing a total of four separate simulations, adding one pulse at a time. The half-width of each succeeding pulse was made to coincide with $L_{r e s}$, which was determined by the previous simulation. The results appear in Fig. 11(b).

A feature revealed only when the PIC code is used to model the RLPA is nonlinear Landau damping. Notice that in Fig. 11(b) the wake-field amplitude decreases rapidly after it reaches its peak, after the last of the four laser pulses is added to the train. This is not observed in the single-pulse case of Fig. 11(a), or for the case of the pulse train solved with the fluid-code, Fig. 4(b). The PIC-simulation particles start with a small temperature such that their initial velocities are much less than the phase velocity of the wave. However, electron velocity distributions calculated in at various times during the simulation indicate that the first few pump pulses accelerate some of the background electrons close to the relativistic phase velocity of the wake field, so that these electrons are in the right phase to give energy to the wave. Besides decreasing the amplitude of the wave after it reaches its peak, damping also undoubtedly competes with the latter pump pulses, reducing the rate of growth of the plasma wave.

\section{PULSE TRAIN GENERATION TECHNIQUES}

There are several ways of producing the required pulse train in practice. The first, shown in Fig. 10, is to use Fourier filtering. In this case, a mask is placed in the pulse stretcher of a chirped pulse amplification system ${ }^{8}$ to modulate the beam in such a way that, when it is recompressed, a series of pulses with arbitrary spacings and widths will be produced ${ }^{29}$. The minimum rise time of each individual pulse is still governed by the gain bandwidth of the amplifiers. Shaped pulses have in fact been amplified in the laboratory ${ }^{30}$. Another possibility is to use several separate compressors with adjustable lengths and delays. Finally, pulse shaping is much more versatile than optical mixing of two laser lines, as is used in the PBWA, because in the latter case the limited choice high gain laser lines severely restricts the choice of operating plasma density. 


\section{CONCLUSIONS}

Optimal pulse widths and interpulse spacings were exactly computed from analytical theory for a train of square pulses, and were optimized numerically for a train of sine pulses with realistic rise times. A resonant region of the plasma wave phase space was found where the plasma wave is driven by the laser most efficiently. The width of this region, and thus the optimal finite-rise-time laser pulse width, was found to decrease with increasing density and plasma wave amplitude. However, at low densities, where electron energy gain is greatest, all pulses could be optimized up to the wave-breaking amplitude for the realistic trains considered in this paper. Thus when compared to the PBWA and LWFA, the RLPA is found to drive nonlinear plasma waves with superior energy efficiency. The sensitivities of the wake field to changes in the plasma density and laser intensity were not found to pose significant technological problems. Wake fields from trains with somewhat-longer-than-optimal pulse widths were found to be considerably less sensitive to variation of interpulse spacing without sacrificing much efficiency. PIC code results validated the use of the quasi-static approximation and the assumption of nonevolving pulse shapes in the fluid code for the short distances studied. They also showed the importance of nonlinear Landau damping at late times. The RLPA was shown to be superior for electron acceleration to either the PBWA of the LWFA because comparable plasma wave amplitudes may be generated at lower plasma densities, thus reducing electron-phase detuning, or at lower laser intensities, thus reducing laser-plasma instabilities. Finally, it was shown that practical methods for tailoring laser pulse trains using pulse-shaping techniques are currently available.

\section{ACKNOWLEDGEMENTS}

This work was supported by NSF and DoE. The authors acknowledge useful conversations with X. Liu, J. Squier, G. Mourou, J. Krall, A. Ting, P. Sprangle, and W. Mori.

\section{REFERENCES}

1. Eric Esarey's home institution: Beam Physics Branch, Naval Research Laboratory, Washington, DC 20375, (202) 404-7720.

2. See e.g., Advanced Accelerator Concepts, ed. by J. S. Wurtele, AIP Conf. Proc. 279 (American Institute of Physics, New York, 1993).

3. T. Tajima and J. M. Dawson, Phys. Rev. Lett. 43, 267 (1979).

4. C. Clayton, et al., Phys. Rev. Lett. 54, 2343 (1985); Y. Kitigawa, et al., Phys. Rev. Lett. 68, 48 (1992); F. Amiranoff, et al., Phys. Rev. Lett. 68, 3710 (1992); H. Hamster, et al., Phys. Rev. Lett. 71, 2725 (1993).

5. A. I. Akhiezer and R. V. Polovin, Sov. Phys. JETP 3, 696 (1956).

6. J. M. Dawson, Phys. Rev. 113, 383 (1959). 
7. L. M. Gorbunov and V. I. Kirsanov, Sov. Phys. JETP 66, 290 (1987); P. Sprangle, et al., Appl. Phys. Lett. 53, 2146 (1988).

8. P. Maine, et al., IEEE J. Quantum Electron. 24, 398 (1988); G. Mourou and D. Umstadter, Phys. Fluids B 4, 2315 (1992).

9. P. Sprangle, et al., Phys. Rev. Lett. 69, 2200 (1992); J. Krall, et al., Phys. Rev. E 48, 2157 (1993); E. Esarey, et al., Phys. Fluids B 5, 2690 (1993).

10. N. E. Andreev, et al., JETP Lett. 55, 571 (1992); T. Antonsen, Jr. and P. Mora, Phys. Rev. Lett. 69, 2204 (1992).

11. E. Esarey, J. Krall and P. Sprangle, Phys. Rev. Lett. 72, 2887 (1994).

12. W. P. Leemans, et al., Phys. Rev. A 46, 1091 (1992); P. Monot, et al., J. Opt. Soc. Am. B 9, 1579 (1992).

13. E. Esarey and M. Pilloff, "Maximum Energy Gain in a Nonlinear Plasma Wave," Proceedings of Workshop on Advanced Accelerator Concepts, Lake Geneva, WI, 1994, Advanced Accelerator Concepts, ed. by P. Schoessow (American Institute of Physics, New York, to be published).

14. D. Umstadter, E. Esarey, and J. Kim, Phys. Rev. Lett. 72, 1224 (1994).

15. V. I. Berezhiani and I. G. Murusidze, Physica Scripta 45, 87 (1992).

16. G. Bonnaud, D. Teychenne and J. L. Bobin, Phys. Rev. E 48, 3248 (1993); Phys. Rev. E 50, 36 (1994); S. Dalla and M. Lontano, Phys. Rev. E 49, 1819 (1994); Phys. Lett. A 173, 456 (1993); K. Nakajima, Phys. Rev. A 45, 1149 (1992).

17. C.G. Durfee, III and H.M. Milchberg, Phys. Rev. Lett. 71, 2409 (1993).

18. D.L. Fisher and T. Tajima, Phys. Rev. Lett. 71, 4338 (1993).

19. J. V. Rudd et al., Opt. Lett. 18, 2044 (1993); J. P. Zhou, et al., Opt. Lett. 19, 126 (1994); C. P. Barty et al., "Ultrashort pulse multiterawatt Ti:sapphire laser system," in Ultrafast Phenomena, Vol. 7, 1994 OSA Technical Digest Series (Optical Society of America, Washington, DC, 1994), p. 324.

20. E. Esarey, et al., IEEE Trans. Plasma Sci. PS-21, 95 (1993).

21. C. D. Decker and W. B. Mori, Phys. Rev. Lett. 72, 490 (1994).

22. Current laser technology has achieved a maximum intensity of $I=5.0 \times 10^{18} \mathrm{~W} / \mathrm{cm}^{2}$.

23. For square pulses, since $\tau_{o p t}$ increases with $x_{\max }$, the appropriate comparison is at fixed $n_{e 0}$; and, in the low- $n_{e 0}$ regime, square pulses with finite rise times $\tau_{\text {rise }} \simeq \tau_{\min } / 2$ behave as ideal square pulses, provided $\tau_{\text {rise }}<L_{\text {res }} / 2 c$ for all the pulses in the train.

24. C. Tang, P. Sprangle, and R. Sudan, Appl. Phys. Lett. 45, 375 (1984). 
25. For a single pulse with duration $\tau$ less than $10 \mathrm{ps}$, the heat diffusion time, the damage threshold of the last laser grating of a chirped pulse amplification system depends only on total laser fluence. For longer pulses, the damage threshold fluence increases as $\tau^{1 / 2}$. [See e.g., D. Du, et al., Appl. Phys. Lett. 64, 3071 (1994).] The damage threshold of a pulse train with picosecond interpulse spacings behaves similarly, where $\tau$ in this case would be the duration of the pulse train.

26. S. C. Wilks, J. MDawson, and W. B. Mori, Phys. Rev. Lett. 61, 337 (1988); V. A. Mironov, A. M. Sergeev, E. V. Vanin, and G. Brodin, Phys. Rev. A 42, 4862 (1990); E. Esarey, G. Joyce, and P. Sprangle, Phys. Rev. A 44, 3908 (1991).

27. A. E. Dangor, et al., IEEE Trans. Plasma Sci. PS-15, 161 (1987).

28. D. R. Nicholson, Introduction to Plasma Theory (Krieger, Malabar, FL, 1992).

29. A. M. Weiner, et al., IEEE J. Quantum Electron. 28, 908 (1992).

30. Y. H. Chuang, Z. W. Li, D. D. Meyerhofer, and A. Schmid, Opt. Lett. 16, 7 (1991).

\section{FIGURES}

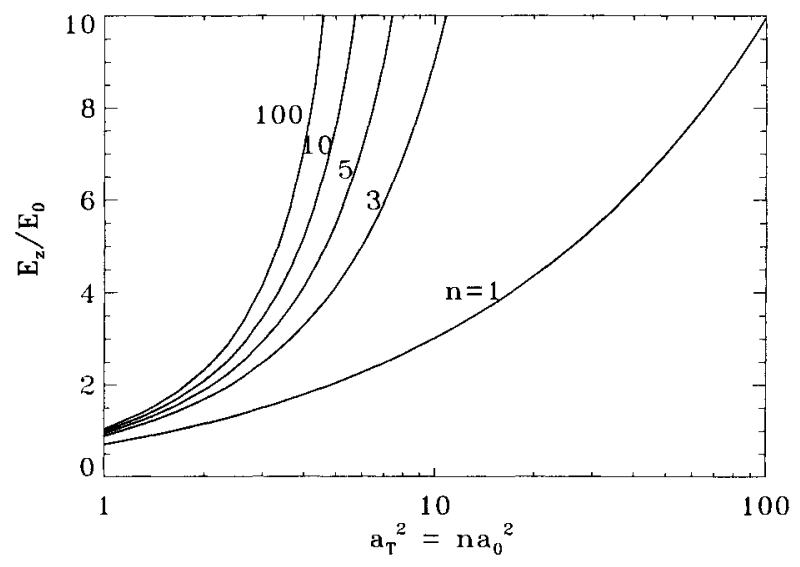

Figure 1: The maximum electric field $\left(\hat{E}_{\max }\right)$ vs the quantity $a_{T}^{2}=n a_{0}^{2}$ for $n=1,3,5,10$ and 100 . 


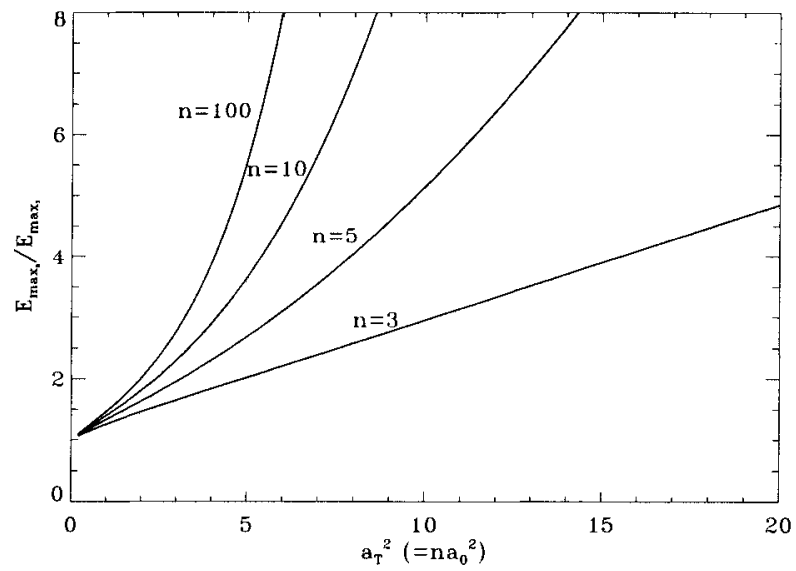

Figure 2: The ratio of the maximum field achieved with a train of pulses $\left(\hat{E}_{\text {max }_{n}}\right)$ over that achieved with a single pulse $\left(\hat{E}_{\max }\right)$ of the same energy versus the quantity $a_{T}^{2}=n a_{0}^{2}$ for $n=3,5,10$ and 100 .

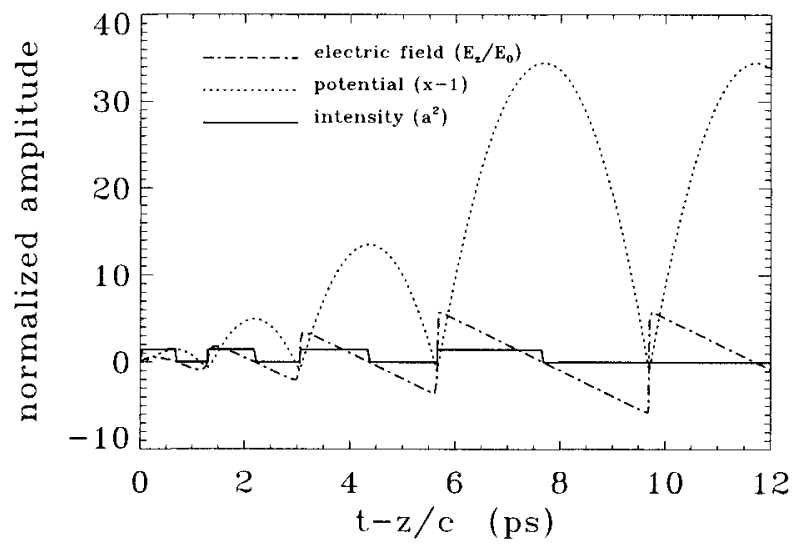

Figure 3: Numerical solutions for an optimized square pulse train at $n_{e}=10^{16} \mathrm{~cm}^{-3}$ and with $a_{0}=1.2$. 

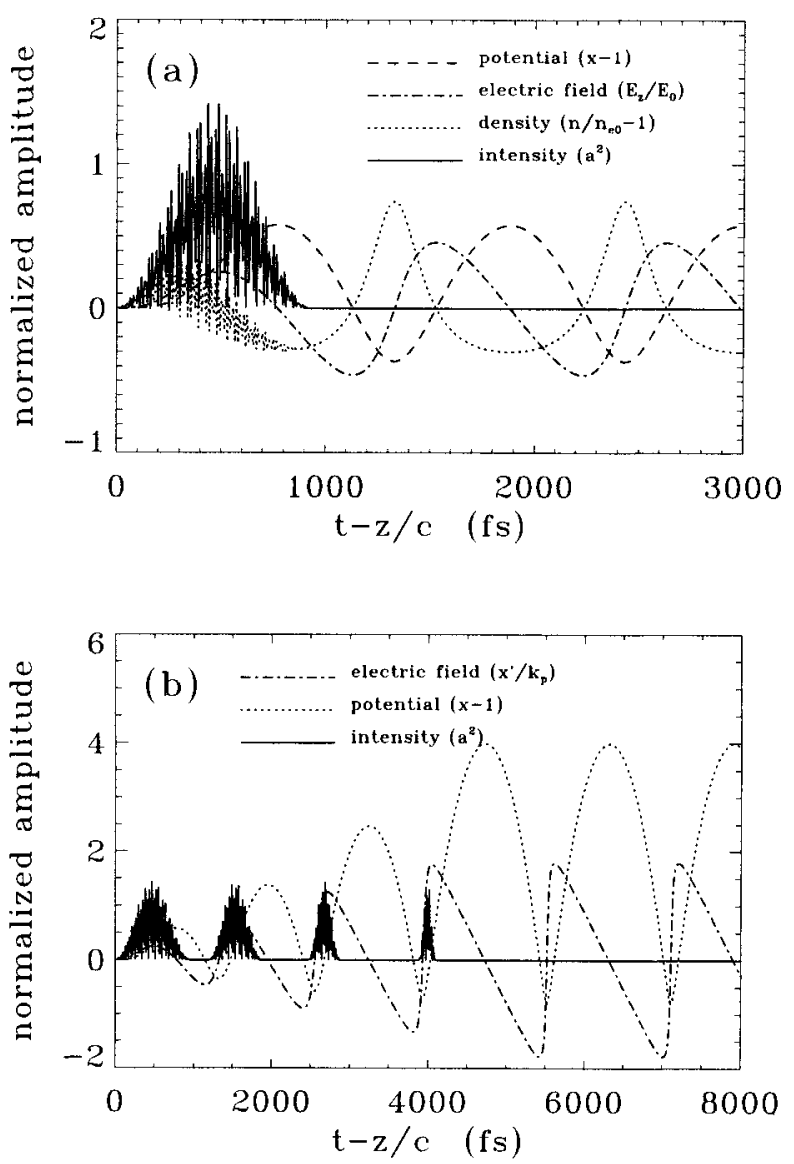

Figure 4: Numerical solutions for LWFA and RLPA with sine-shaped pulses: (a) single sine pulse at $n_{e}=10^{16} \mathrm{~cm}^{-3}$ with $a_{0} \simeq 2$, and (b) an optimized sine pulse train at $n_{e}=10^{16} \mathrm{~cm}^{-3}$ with $a_{0}=1.2$. 

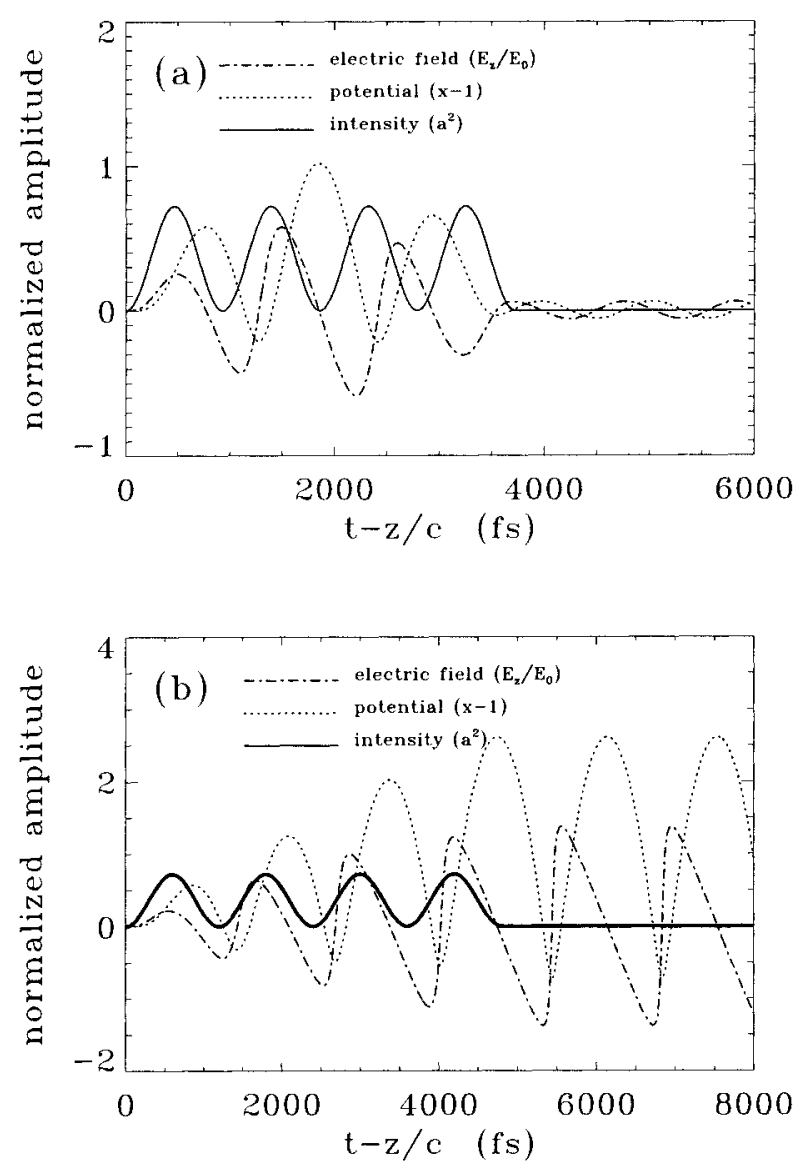

Figure 5: Numerical solutions for the PBWA: (a) without optimization, showing the effects of detuning, and (b) with optimization. 


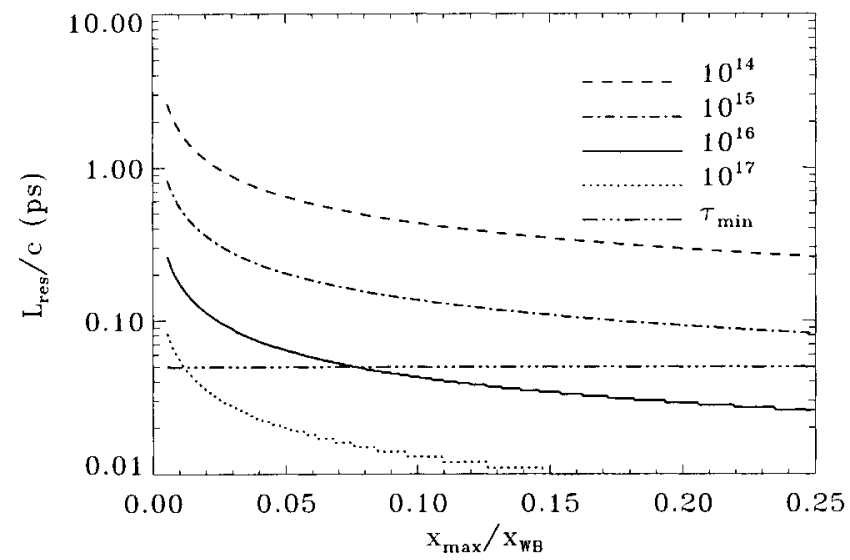

Figure 6: Plot of $L_{r e s} / c$ vs $\varepsilon=E_{z} / E_{W B}$ for various densities. Finite rise-time effects are important for $L_{\text {res }} / c<\tau_{\text {min }}$. 

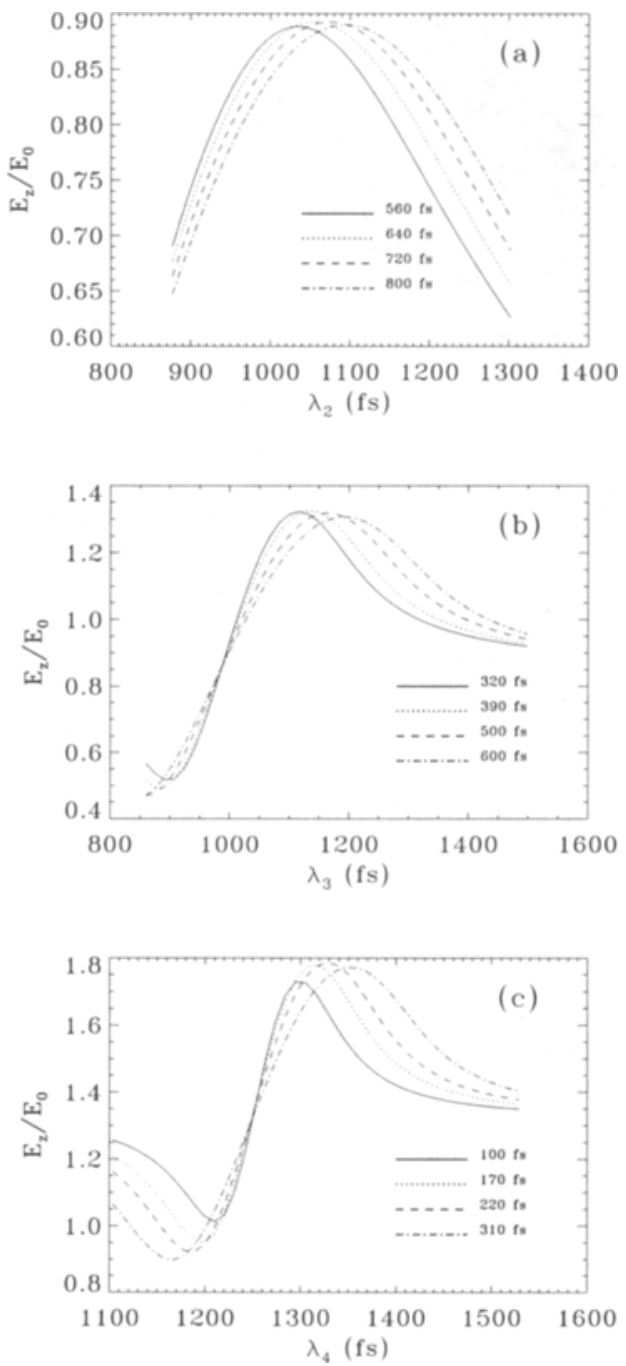

Figure 7: The maximum electric field $\hat{E}_{\max }$ produced by varying both the pulse widths $\tau$ and interpulse spacings $\lambda_{N_{n}}$, for the second $n=2$ (a), third $n=3$ (b), and fourth $n=4$ (c) pulses. Note the change in scaling of $\hat{E}_{\max }$ for the three plots. 

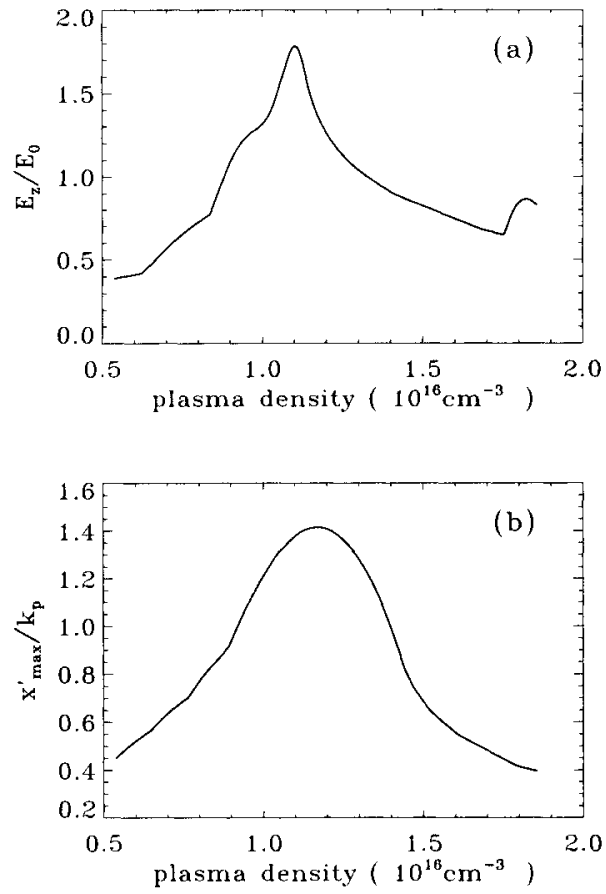

Figure 8: Final wake-field amplitude as a function of the value of the initial plasma density for: (a) RLPA, and (b) PBWA. The arrow indicates the density corresponding to the resonant density in the linear approximation, $\Delta \omega=\omega_{p}\left(n_{e}\right)$. 

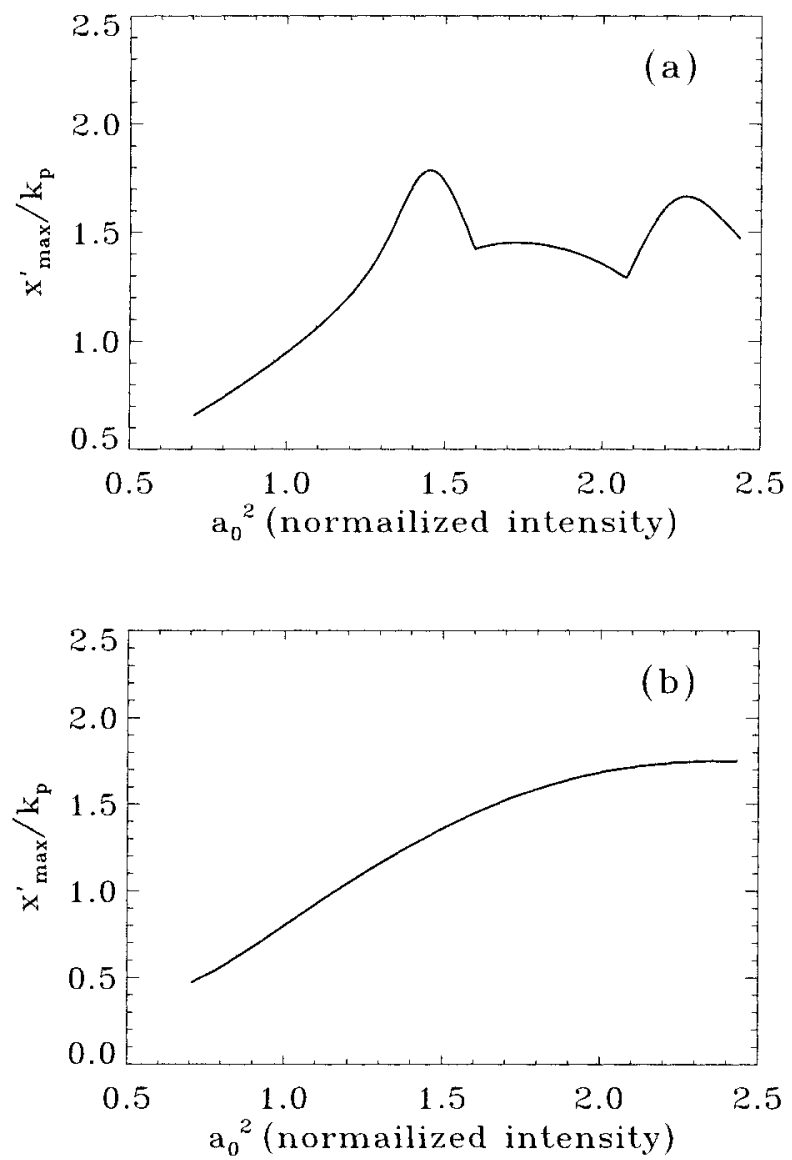

Figure 9: Final wake-field amplitude as a function of laser intensity for (a) RLPA, and (b) PBWA. 


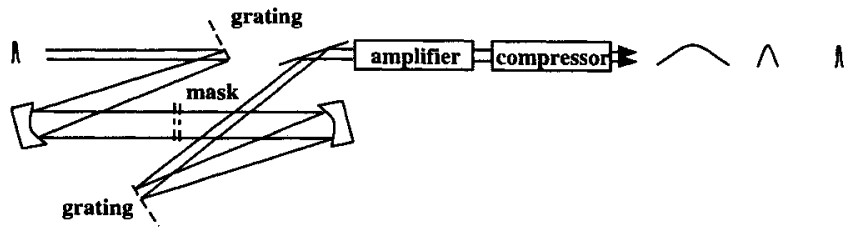

Figure 10: A variably spaced pulse train with arbitrary pulse widths is produced by use of Fourier filtering in the laser stretcher stage. 

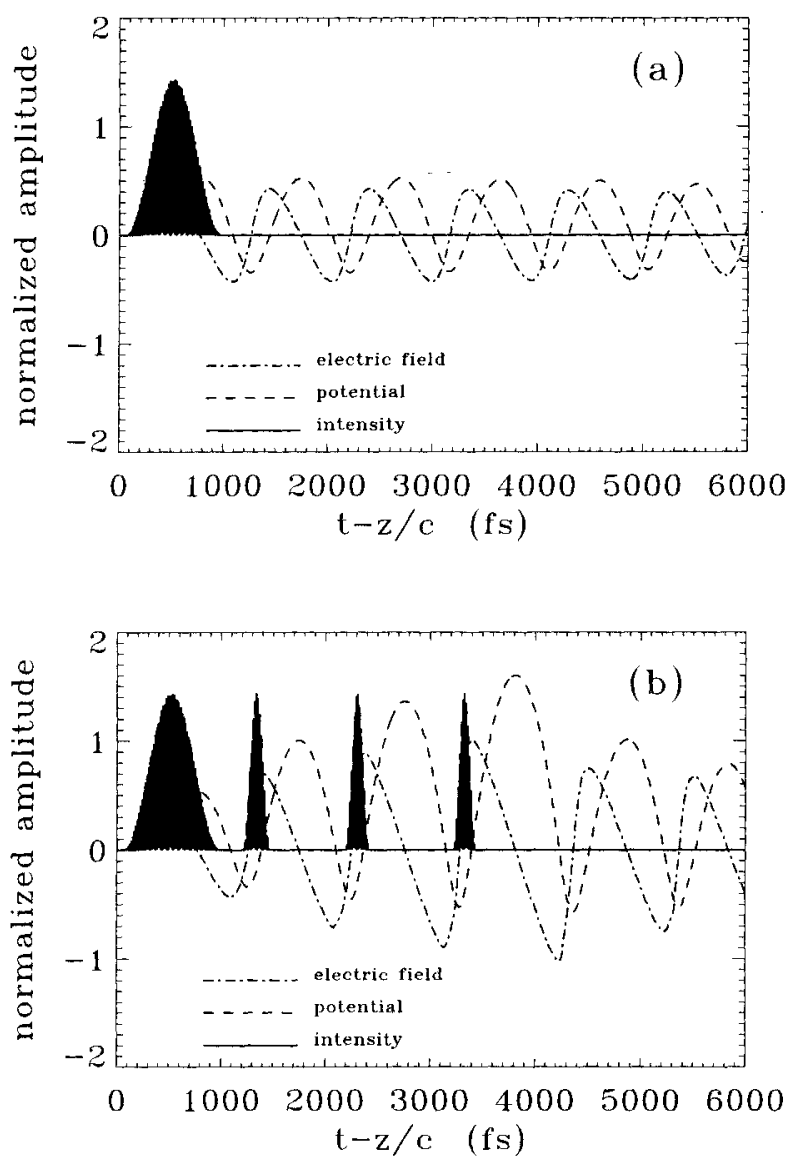

Figure 11: PIC simulation solution with $n_{e}=10^{16} \mathrm{~cm}^{-3}$, and $a^{2}=1.44$ for (a) a single sine pulse, and (b) four sine pulses. Plasma wave damping is observed at late times only in the latter case. 